\title{
Nanotecnología, una alternativa para mejorar la calidad del agua
}

\author{
Silvia Lucila Gelover Santiago*
}

RESUMEN: La curiosidad, creatividad e ingenio, característicos de la especie humana, encuentran un paraíso al explorar mundos inimaginables, tal y como es el nanomundo. Tamaños microscópicos, formas exóticas y sobre todo fenómenos y mecanismos que sólo suceden a ese nivel constituyen un universo que ofrece nuevas posibilidades en cuanto a la aplicación de nanomateriales como posibles soluciones a los problemas de contaminación que cotidianamente enfrentamos. En este artículo se presenta una visión sobre las posibilidades de aplicación de nanomateriales como alternativa para mejorar la calidad del agua, especialmente cuando están presentes contaminantes orgánicos y microbiológicos.

PALABRAS CLAVE: nanocatalizadores, $\mathrm{TiO}_{2}$, fotocatálisis solar, tratamiento del agua con nanomateriales, desinfección fotocatalítica.

ABSTRACT: Curiosity, creativity and human wit, characteristics of mankind, find a paradise exploring unimaginable worlds, as is the nanoworld. Microscopic sizes, shapes and especially exotic phenomena and mechanisms that only happen at this level constitute a universe that offers new possibilities for the application of nanomaterials as alternative solutions to pollution problems that we face daily. This article presents a vision of the possibilities of application of nanomaterials as an alternative to improve water quality, especially when organic and microbiological contaminants are present.

KEY WORDS: Nanocatalysts, $\mathrm{TiO}_{2}$, solar photocatalysis, nanomaterials water treatment, photocatalytic disinfection.

\section{El agua, un tesoro en riesgo}

La nanotecnología es una nueva opción científica y tecnológica para mejorar nuestro ambiente.

En lo que respecta al agua, es bien sabido que este maravilloso recurso se encuentra con preocupantes limitantes para el uso del ser humano.

Las propiedades del agua hacen de ella una sustancia maravillosa, su poder disolvente representa a la vez una ventaja y una desventaja. Sus inusuales propiedades físicas, químicas y esa, tan especial, capacidad de formación de puentes de hidrógeno la hacen sumamente especial. Representa el crisol en donde se gestó la vida, el hogar de especies que regulan importantes ciclos biogeoquímicos. Receptáculo natural de infinidad de sustancia, se ha convertido, también, en el destino final de muchos de nuestros desechos.

* Instituto Mexicano de Tecnología del Agua. Paseo Cuauhnáhuac 8532. Progreso, Jiutepec, Morelos México. 62550. Correspondencia: (sgelover@tlaloc.imta.mx). 
El ciclo del agua hace de ella el medio para el transporte de nutrientes, sales, gases y detritus. Y también, un medio de propagación de enfermedades: ha representado un vector de padecimientos, como el cólera y diversas afecciones gastrointestinales causadas por virus, bacterias y otros microrganismos patógenos que, aún hoy, son la causa de muchas muertes, sobre todo infantiles. Esto es particularmente doloroso en países como el nuestro en donde, debido a la pobreza y aislamiento en que aún viven millones de personas, resulta muy difícil el acceso a agua de buena calidad, situación que se agrava para las zona de alta sequía (como el norte del país) en donde, incluso el acceso a este recurso es muy limitado, sin importar su calidad.

La pequeña fracción de agua dulce de que disponemos para nuestras necesidades, se ve amenazado por situaciones de muy diversa naturaleza, como son el cambio climático que incrementa los problemas de intrusión salina, y la contaminación causada por las actividades humanas.

Son tres las grandes necesidades de nuestra actual sociedad: comida, agua y energía. Y visto de otra manera éstas tres son agua: 1) para la agricultura, ganadería e industria alimentaria; 2) agua como recurso en sí mismo, y, 3) agua como un insumo para obtener las crecientes cantidades de energía de las fuentes convencionales.

El progreso y la sofisticación de los productos químicos que hemos incorporado al ambiente es tal que impide el funcionamiento de los sistemas naturales de depuración así como de nuestros mismos sistemas de tratamiento de aguas residuales industriales y municipales. Ha llegado el momento en que los sistemas de depuración tradicionales, basados en sistemas biológicos, resultan insuficientes.

\section{Los nanomateriales, una opción de tratamiento}

Así que nos hemos visto obligados a emplear métodos alternativos, los llamados procesos avanzados de oxidación son quizá el ejemplo más evidente. En adición a esto, los tratamientos de purificación y desinfección del agua han encontrado en los nanomateriales alternativas no previstas hasta hace poco tiempo. Y el uso de materiales nanoparticulados, iniciado en la década de los años 70 se ha desarrollado a un ritmo exponencial.

En su artículo sobre nanotecnología, Theron y colaboradores (2008) dicen de la misma que "es la ingeniería y el arte de manipular la materia a nanoescala, es decir, entre 1 y 100 nanometros", y presentan un panorama del potencial de utilizar nuevos materiales para el tratamiento de agua (superficial, subterránea o bien de aguas subterráneas que se hallen contaminadas), para la detección de sustancias disueltas en muy baja concentración, o bien para la desinfección.

El tratamiento del agua se puede visualizar en dos grandes esferas, el tratamiento del agua para su potabilización y el tratamiento de las aguas 
residuales. Los contaminantes que es necesario remover del agua son de manera genérica metales, agentes inorgánicos, agentes orgánicos y microrganismos patógenos.

\section{Metales y metaloides disueltos, riesgos y alternativas de tratamiento}

En relación al tratamiento del agua potable, eliminar la presencia de metales como el cromo, cadmio, cobalto, mercurio, zinc y cobre, tiene relevancia por la toxicidad que tienen. Lo mismo sucede con el arsénico, metaloide que, a diferencia de los metales mencionados se encuentra en las fuentes de abastecimiento de agua potable, de manera muy importante, debido a una contaminación natural, es decir, a que el agua subterránea adquiere esta sustancia al estar en contacto con minerales que lo contienen. Países en todo el mundo enfrentan el reto de eliminar arsénico de sus aguas subterráneas pues la ingesta de este metaloide causa problemas de salud afectando varios órganos en diferentes niveles (piel, glóbulos rojos, médula, hígado, puede causar cáncer en piel, riñones y vejiga) alterando así el bienestar de la población. En su revisión, Sharma et al., (2009) presentan un panorama acerca de los avances que se tienen en la aplicación de - como ellos llaman a los nanomateriales-, "estos exóticos materiales" en la remediación de agua.

La técnica tradicional para remover metales del agua es la adsorción. El adsorbente por excelencia es el carbón activado. Este material encuentra su símil en el mundo microscópico en los nanotubos de carbono (CNTs, por sus siglas en inglés). Estas nanoestructuras fueron reportadas inicialmente en 1991, hace poco más de veinte años. Ya sea en forma de nanotubos de una sola o múltiples paredes, estos materiales presentan propiedades únicas tales como su alta estabilidad química y gran área superficial, por lo que múltiples trabajos han tenido como objetivo investigar su aplicación en el tratamiento de aguas residuales conteniendo iones metálicos. Los nanotubos de carbono además de adsorbentes pueden ser soportes para otros materiales adsorbentes.

El empleo de nanopartículas de hierro zerovalente (nZVI, por sus siglas en inglés) en la remediación de sitios contaminados con cromo, nitratos, percloratos o con disolventes organoclorados es otro ejemplo de la aplicación de estos materiales para remediar el agua subterránea. Durante los últimos 15 años, se ha investigado el uso de hierro metálico nanoparticulado como una nueva herramienta para el tratamiento de agua y suelo contaminados. La tecnología ha alcanzado un estatus comercial en muchos países alrededor del mundo; sin embargo, aún debe obtener una aceptación más amplia (Crane y Scott, 2012). Los factores clave a atender incluyen: la preocupación por su destino a largo plazo, la transformación y la ecotoxicidad de nZVI en sistemas ecológicos, la falta de estudios comparables para diferentes materiales $\mathrm{nZVI}$ y estrategias de implementación. Algunas investigaciones han 
puesto de relieve nuevas preocupaciones, como la posibilidad de la recuperación de la movilidad de los metales pesados y radionúclidos después de periodos prolongados, así como la importancia fundamental de ser capaces de predecir, con exactitud, los procesos a largo plazo en los aspectos físicos, químicos y en el destino biológico de los sitios contaminados tras el tratamiento con nZVI.

\section{Contaminantes orgánicos}

Con respecto a la presencia de contaminantes orgánicos, el abanico es muy amplio pues se pueden encontrar en el agua desde plaguicidas, producto de las actividades agrícolas y domésticas; residuos de fármacos y productos de cuidado personal, algunos de los cuales causan alteraciones en el sistema endocrino, por lo que son llamados disruptores endocrinos. También es posible encontrar una amplia variedad de productos químicos empleados en la industria: disolventes, plastificantes, colorantes, subproductos de la combustión, subproductos de la cloración; en fin, un mar de compuestos que contaminan nuestras aguas.

Tradicionalmente, las metodologías utilizadas en el tratamiento del agua incluyen procesos como coagulación, floculación, sedimentación, filtración, adsorción y oxidación. Sin embargo, en ocasiones estas tecnologías resultan insuficientes para lograr la remoción de los contaminantes orgánicos, por lo que aún en los efluentes de plantas de tratamiento es posible detectar la presencia de estos compuestos.

\section{Nanomateriales, características, funciones y mecanismos}

La variedad de nanomateriales ahora disponible cubre diversas posibilidades de tratamiento. Muchos de los tratamientos convencionales se han visto mejorados por la incorporación del uso de nanomateriales. Los tratamientos convencionales de adsorción, oxidación y separación se ven hoy potenciados y han sido transformados por el uso de nanosorbentes, nanocatalizadores y nanopartículas bioactivas, gracias a las características únicas que les imparte su tamaño, como son una gran área superficial por unidad de volumen, significativas propiedades físicas y químicas y efectos cuánticos que ocurren sólo a nanoescala. La disminución en el tamaño de partícula tiene dos beneficios significativos. Por un lado la alta densidad superficial de sitios activos disponibles para la adsorción de sustratos, consistente con la alta relación área superficial/volumen típica de las nanopartículas, hace posible incrementar la velocidad de las fotorreacciones. Por otro lado, para el caso de los semiconductores la energía de brecha depende de efectos cuánticos, lo que permite el diseño de materiales con energías de brecha seleccionadas, y con ello de propiedades redox específicas. A través de la síntesis de nanocristales de alta calidad también se logra una máxima separación del par hueco-electrón. 
De los nanocatalizadores empleados en el tratamiento de agua, el más utilizado es y ha sido el dióxido de titanio, también conocido como titania. La titania existe en tres formas cristalinas: anatasa, rutilo y brookita, además de su fase amorfa. Sólo las dos primeras tienen utilidad como fotocatalizadores.

Los trabajos en cuanto al uso del dióxido de titanio para la eliminación de contaminantes tanto biológicos como químicos crece día con día y la variedad de sustancias y entes biológicos que son abordados por estas metodologías también crece.

\section{Nanomateriales y fotocatálisis solar}

Una de las vertientes es el uso de dióxido de titanio nanoparticulado en combinación con energía solar, en donde se aprovecha un recurso natural, inagotable para fines prácticos y disponible para una amplia zona del planeta, con una novedosa variedad de materiales denominados nanocatalizadores. El dióxido de titanio presenta propiedades que lo hacen un material ciertamente relevante. Es un material relativamente abundante, por lo que su costo resulta conveniente, no es tóxico, presenta baja reactividad química, tiene buena fotoestabilidad y es estable en un amplio intervalo de $\mathrm{pH}$, es decir, puede trabajar bien en medios ácidos, pero también en medios básicos. Al activarse con energía UV, genera especies altamente reactivas que son las responsables de su alto poder oxidante, mismo que es aprovechado para realizar la transformación de moléculas que de otra manera sería muy difícil oxidar. Al ser un catalizador, el dióxido de titanio sirve solamente como mediador en las reacciones químicas, sin transformarse, lo que representa una ventaja pues en general se puede reutilizar una y otra vez.

Una de las desventajas, sin embargo, es que la proporción realmente útil del espectro solar para activar a este fotocatalizador es apenas del $5 \%$ en el mejor de los casos, por lo que existe una gran cantidad de esfuerzo encaminado a la modificación del semiconductor de tal suerte que sea posible aprovechar una mayor fracción de la radiación solar.

$\mathrm{Al}$ ser un semiconductor, éste requiere de energía mayor que su energía de brecha para generar el par electrón-hueco, base de su funcionalidad. Bajo la radiación adecuada, el dióxido de titanio experimenta el salto de uno de sus electrones del nivel de valencia al nivel de conducción, dando origen al par hueco-electrón. El hueco captura un electrón de las especies cercanas; probabilísticamente es más posible su reacción con agua al hallarse en un medio acuoso, con lo que se genera una especie denominada radical hidroxilo, es decir, una molécula de agua a la que se le ha abstraído un electrón y que simultáneamente libera un protón (el protón se asocia fácilmente con otras moléculas de agua del medio), en tanto que el radical formado, con un potencial de oxidación muy alto $(2.8 \mathrm{eV})$, apenas por debajo del flúor $(3.03 \mathrm{eV})$, tiende a reaccionar con cualquier especie oxidable presente. Para 
nuestra ventaja muchos de los contaminantes son oxidables por el radical hidroxilo, o bien son oxidados directamente por los huecos cuando estas especies se encuentran adsorbidas sobre el catalizador. En el otro extremo, el electrón, ahora en la capa de valencia, puede dar lugar, al reaccionar con oxígeno, a la formación del radical superóxido $\left(\mathrm{O}_{2} \cdot\right)$, y en presencia de peróxido de hidrógeno al radical perhidroxilo (HOO •). Los radicales así formados reaccionan con moléculas adsorbidas a la superficie del catalizador resultando en la hidroxilación, decloración, oxidación e incluso mineralización de las moléculas orgánicas.

\section{Mecanismo de la fotocatálisis}

De acuerdo con Kamat (1996), los procesos fotoinducidos que siguen a la excitación a través de la brecha energética de un semiconductor se pueden escribir de la siguiente manera: Después de la excitación, los portadores de carga inicialmente libres quedan atrapados en sitios denominados defectos, estos portadores de carga pueden sufrir recombinación radiativa y no radiativa. La acumulación de carga en el diminuto espacio de un semiconductor coloidal es importante porque mejora la eficiencia de las reacciones redox interfaciales. Estudios especializados han mostrado que en la anatasa, los electrones fotogenerados son atrapados en $\mathrm{Ti}^{4+}$ dentro de la masa del semiconductor, en tanto que los grupos hidroxilo básicos sobre la superficie del $\mathrm{TiO}_{2}$, pueden ser considerados trampas profundas para los huecos de la banda de valencia. El hueco queda en los grupos hidroxilo superficiales, por lo que puede reaccionar con las sustancias orgánicas presentes en el medio.

La separación efectiva de las cargas fotogeneradas puede conseguirse, por ejemplo, por medio de la deposición de metales en la superficie del semiconductor (Pradeep, 2009; Tada et al., 2009). La presencia de depósitos metálicos sobre la superficie del semiconductor altera las propiedades eléctricas de éste, puesto que se crean nuevas interfaces de tipo metal-semiconductor.

El uso de fotocatalizadores soportados, con aplicación de potenciales eléctricos permite separar las reacciones anódicas y catódicas, y reducir drásticamente la recombinación electrón-hueco (Jiang et al., 2007). A pesar de sus limitaciones, los procesos fotocatalíticos basados en dióxido de titanio han alcanzado un elevado grado de madurez tecnológica. Si bien el rendimiento cuántico (número de eventos producidos por fotón absorbido) suele ser bajo en términos de conversión química, los rendimientos obtenidos, en términos de conversión en función del tiempo, aprovechando luz solar o iluminación con lámparas, son adecuadamente elevados.

La fotocatálisis solar está fundamentada en la absorción de luz por compuestos semiconductores como el dióxido de titanio, que causan cambios energéticos a nivel molecular, mismos que promueven la generación del radical hidroxilo, la generación de ésta y otras especies relevantes en el proceso de oxidación, se ilustra en la figura 1. 
FIGURA 1. Esquema de los procesos fundamentales en fotocatálisis heterogénea.

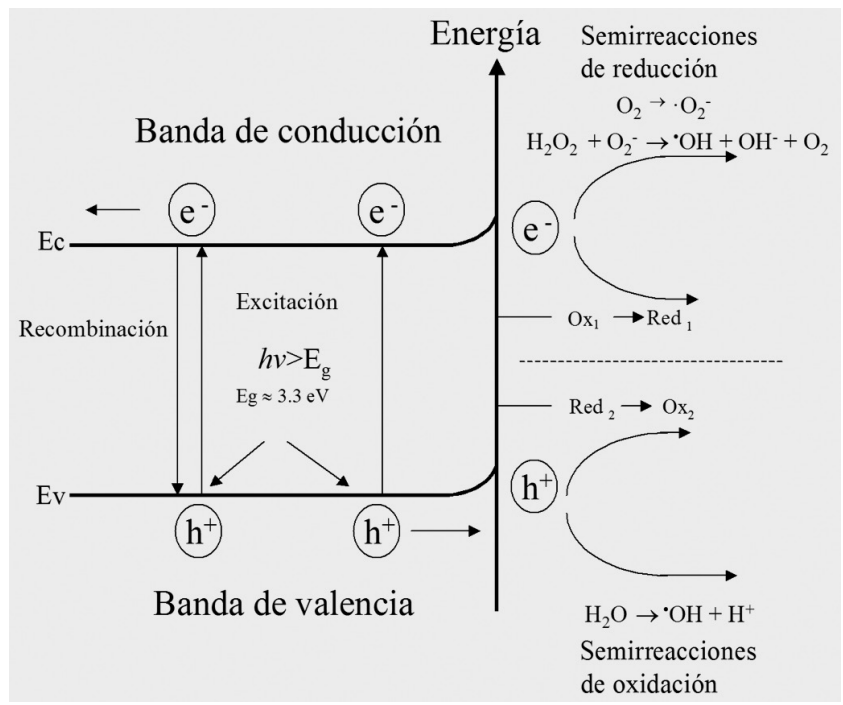

Fuente: Elaboración propia.

Las principales reacciones que ocurren en el sistema son:

Foto-excitación: $\mathrm{TiO}_{2}+h \nu$ e $+\mathrm{h}^{+}$(generación del par electrón-hueco)

Atrapamiento de portadores de carga (electrones): $\mathrm{e}_{C B}^{-} \longrightarrow \mathrm{e}_{\mathrm{TR}}^{-}$

Atrapamiento de portadores de carga (huecos): $\mathrm{h}^{+}{ }_{\mathrm{VB}} \longrightarrow \mathrm{h}^{+}{ }_{\mathrm{TR}}$

Recombinación electrón-hueco: $\mathrm{e}_{\mathrm{TR}}^{-}+\mathrm{h}_{\mathrm{VB}}^{+}\left(\mathrm{h}_{\mathrm{TR}}^{+}\right) \longrightarrow \mathrm{e}_{\mathrm{CB}}^{-}+$calor

Captura de especies fotoexcitadas: $\left(\mathrm{O}_{2}\right)_{\mathrm{ads}}+\mathrm{e}^{-} \longrightarrow \mathrm{O}_{2}{ }^{-}$

Oxidación de hidroxilos: $\mathrm{OH}^{-}+\mathrm{h}^{+} \longrightarrow \cdot \mathrm{OH}$

Fotodegradación de orgánicos (acción de radicales hidroxilo):

$$
\mathrm{R}-\mathrm{H}+{ }^{\bullet} \mathrm{OH} \longrightarrow \mathrm{R}^{\bullet}+\mathrm{H}_{2} \mathrm{O}
$$

Fotodegradación de orgánicos (acción de foto-huecos):

$\mathrm{R}+\mathrm{h}^{+} \longrightarrow \mathrm{R}^{+} \longrightarrow$ Productos de degradación intermedios y finales.

Protonación de superóxidos: $\mathrm{O}_{2}{ }^{{ }^{-}}+{ }^{-} \mathrm{OH} \longrightarrow \mathrm{HOO}$

Captura de e fotogenerados: $\mathrm{HOO}^{-}+\mathrm{e}^{-} \longrightarrow \mathrm{HO}_{2}{ }^{-}$

Formación de $\mathrm{H}_{2} \mathrm{O}_{2}$ : $\mathrm{HOO}^{-}+\mathrm{H}^{+} \longrightarrow \mathrm{H}_{2} \mathrm{O}_{2}$

donde $\mathrm{e}^{-}{ }_{\mathrm{TR}} \mathrm{y} \mathrm{h}^{+}{ }_{\mathrm{TR}}$ representan al electrón atrapado en la superficie de la banda de valencia y al hueco atrapado en la superficie de la banda de conducción, respectivamente.

Evitar la recombinación del par electrón hueco constituye un área de investigación importante en fotocatálisis heterogénea y también para otras 
aplicaciones de semiconductores. La disminución de la recombinación se ha conseguido de diversas maneras: mediante iluminación periódica controlada -dando tiempo a que ocurran procesos limitantes de la velocidad antes de introducir más fotones en el sistema-, a través de la degradación de compuestos orgánicos asistida electroquímicamente (Jiang et al., 2007) en la cual se extraen los electrones fotogenerados del $\mathrm{TiO}_{2}$ mediante la aplicación de una diferencia de potencial entre el ánodo y el cátodo, aumentando de esta manera el tiempo de vida de los huecos o dopando el $\mathrm{TiO}_{2}$ con metales como Ag, $\mathrm{Au}, \mathrm{Cu}, \mathrm{Fe}, \mathrm{Pt}, \mathrm{Sn}, \mathrm{Zn}, \mathrm{Fe}$, o especies con nitrógeno, azufre o carbono, dado que según sus características y concentración, pueden actuar como mediadores de la transferencia de carga interfacial o como centros de recombinación de las cargas fotogeneradas (Choi et al., 1994; Sunada et al.,2003; Zaleska, 2008). Otra aproximación consiste en el uso de materiales mixtos (compositos), como $\mathrm{TiO}_{2} / \mathrm{SnO}_{2} \circ \mathrm{TiO}_{2} / \mathrm{ZnO}$, que permiten una separación eficiente de las cargas fotogeneradas, mediante la acumulación de los electrones en uno de los materiales, y de los huecos en el otro.

De manera alterna, parte de la investigación actual se encamina a modificar el catalizador de tal manera que absorba la radiación visible. Una posibilidad es la fotosensibilización con especies inorgánicas, como el cloruro de platino IV (Tada et al., 2009), o el nitrógeno, el cual ocupa sitios intersticiales y disminuye el salto de banda del $\mathrm{TiO}_{2}$. También es posible modificar la superficie del $\mathrm{TiO}_{2}$ por adsorción de moléculas orgánicas capaces de absorber luz de la región visible del espectro, a este proceso se denomina sensibilización.

\section{Preparación y ejemplo de uso de fotocatalizadores}

Las nanopartículas de la forma comercial más utilizada de $\mathrm{TiO}_{2}$, hasta hace poco Degussa P-25, ahora Evonik, son difíciles de separar una vez que se han utilizado. Con un tamaño de partícula de aproximadamente $30 \mathrm{~nm}$, no se pueden separar del agua por métodos simples como decantación. Se requiere emplear energía, es decir, es necesario centrifugar o realizar una filtración utilizando un tamaño de poro cerrado. Esto justifica que se busque la posibilidad de inmovilizar a las nanopartículas.

Numerosas técnicas han sido desarrolladas para lograr el depósito de películas uniforme y firmemente adheridas a diversas superficies. Entre ellas están: el rocío pirolítico (Martínez et al., 2003), el bombardeo iónico por magnetrón de Radio Frecuencia en inglés RF magnetron sputtering, el depósito por centrifugación (spin coating), el depósito sol-gel por inmersión o dip coating, el depósito electroforético, y el depósito en capa atómica (Atomic Layer Deposition), entre otros. Varias de estas técnicas requieren de equipos sofisticados: cámaras de vacío, aceleradores electromagnéticos y otros que implican altos costos y personal altamente capacitado para la preparación de estos materiales. 
El sistema dip coating ofrece un alternativa relativamente simple y económica para preparar películas por inmersión. Para ello, se prepara un sol con un precursor orgánico de titanio, generalmente un alcóxido, que es hidrolizado para generar una mezcla susceptible de ser depositada en una superficie conveniente, tal como un vidrio de borosilicato tipo Pyrex ${ }^{\circledast}$ con bajo contenido de hierro, que resulta ventajoso al ser un material transparente a buena parte de la radiación solar (Gelover et al., 2004). Para el depósito se utiliza un sistema que pueda retirar el sustrato a depositar a una rapidez constante y controlada. La velocidad con que se retira el sustrato del sol determina el espesor de la película formada. El espesor es una función de la raíz cuadrada de la velocidad de extracción de la película por lo que se recomienda utilizar una velocidad baja de extracción de los sustratos, para obtener películas delgadas.

Una desventaja de trabajar con $\mathrm{TiO}_{2}$ inmovilizado es que se encuentran restringidas, hasta cierto punto, las cantidades del catalizador que se pueden manejar en un sistema determinado. Sin embargo, lo práctico que resulta el manejo del material inmovilizado debe ser valorado en su justa dimensión, pues de esta manera el sistema no genera lodos o residuos que después requieran algún tratamiento y el sistema fotocatalítico puede en principio trabajar de manera continua o al menos semicontinua.

A continuación se muestra el esquema típico de un sistema fotocatalítico en el que se emplea luz solar para el tratamiento de agua contaminada con sustancias no biodegradables. En este caso dentro del fotorreactor se ha colocado el fotocatalizador inmovilizado, de otra manera dentro de él se tendría una suspensión, típicamente lechosa del catalizador Degusa-P25, o alguna otra forma inmovilizada del fotocatalizador.

El sistema consta de un circuito hidráulico que permite el tratamiento de lotes de $20 \mathrm{~L}$ de agua. Es un sistema autónomo (figura 2), provisto de una celda solar que provee la energía necesaria para bombear el fluido desde el depósito que contiene el agua a tratar hacia los tubos fotorreactores. En este caso se empleó una forma inmovilizada de dióxido de titanio sobre pequeños cilindros de tubo Pyrex ${ }^{\circledR}$ (figura 3). Dentro de los tubos se colocó una varilla de vidrio sobre la cual se sujetaron los cilindritos de vidrio recubiertos con el catalizador, esto fue necesario ya que el agua podía arrastrar los cilindritos provocando su acumulación hacia los extremos de los tubos.

Los tubos se encuentran colocados en el centro de una involuta construida con aluminio. La superficie metálica refleja los rayos del sol incidentes. La apertura de la involuta permite tener una concentración solar de uno cuando se coloca inclinada en dirección norte-sur tantos grados como sea la latitud del sitio en que se trabaja.

Como ya se expuso, para la aplicación práctica del dióxido de titanio en el tratamiento de agua, se requiere algo más que el nanocatalizador, se requiere de un colector solar o fotorreactor y ello implica la selección o preparación de materiales reflejantes adecuados para la colección de fotones, 
FIGURA 2. Esquema de un sistema fotocatalítico empleado durante pruebas de degradación de compuestos no biodegradables (por ejemplo fármacos o plaguicidas).

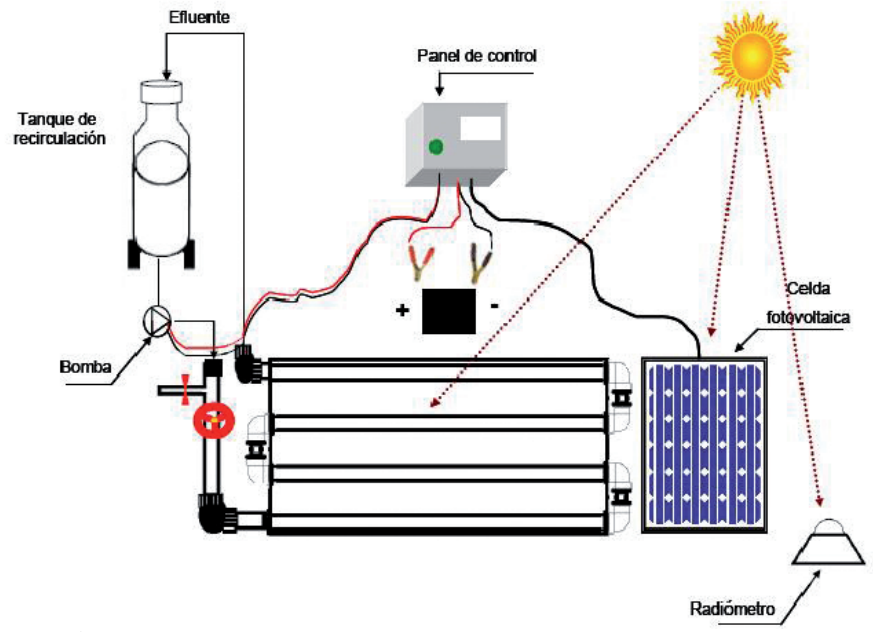

Fuente: Elaboración propia.

materiales suficientemente transparentes que permitan pocas pérdidas para el máximo aprovechamiento de la energía solar, además de resolver el problema de la recuperación del nanocatalizador. Como se ve, hay muchos aspectos prácticos que deben resolverse y aunque hay muchos grupos alrededor del mundo en la búsqueda de soluciones, lo cierto es que esta opción

FIGURA 3. Fotografía de los tubos fotorreactores, se aprecian en el interior los anillos de vidrio, soporte del catalizador. Arriba detalle del arreglo de los anillos.
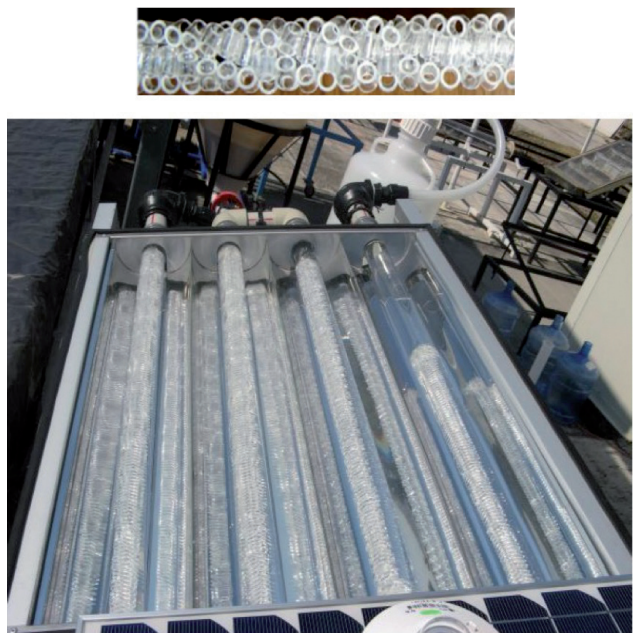

Fuente: Elaboración propia. 
tecnológica aún presenta retos que no serán superados fácilmente en el corto plazo. Esto debe ser una motivación para las jóvenes generaciones que en poco tiempo enfrentarán problemas más agudos que los actuales.

De manera resumida se puede afirmar que las principales limitaciones para el uso generalizado del dióxido de titanio en el tratamiento de agua son:

1. La baja porción de luz UV en el espectro solar de la luz que llega a la superficie de la Tierra.

2. La dificultad operativa del uso del fotocatalizador en forma de polvo, ya que no es fácil de separar del líquido.

3. La alta tendencia de los electrones fotoexcitados de regresar a su estado basal conocida como recombinación.

4. Los altos costos que aún tiene esta opción tecnológica para su aplicación cotidiana.

\section{Desinfección fotocatalítica}

Otra relevante aplicación de los nanomateriales es la desinfección. Métodos tradicionales como la cloración conllevan la formación de subproductos tóxicos, por lo que es deseable hallar métodos de desinfección alternos. En nuestro laboratorio se tuvo una experiencia exitosa cuando se aplicó un material inmovilizado para la desinfección de agua proveniente de un manantial contaminada con microrganismos coliformes fecales y totales (Gelover et al., 2006).

En la literatura se pueden encontrar trabajos sobre la destrucción de diversos microrganismos mediante nanomateriales. Se ha abordado la destrucción de bacterias como E. coli y Enterobacter cloacae (Ede et al., 2012), protozoarios (Huevos de helminto; Leal et al., 2006. Esporas de Clostridium perfringens, Dunlop et al., 2008), y otros microrganismos incluyendo hongos microscópicos, quistes (Lonen et al., 2005) e incluso virus (Sang et al., 2007, Liga et al., 2013).

Pero éste es sólo un pequeño ejemplo de los múltiples trabajos que se han publicado, en donde se han realizado pruebas para inactivar virus, bacterias, y otros organismos patógenos presentes en el agua (Theron et al., 2008).

Como comentario final se puede decir que basta revisar la literatura para darnos cuenta de la variedad de estudios que se desarrollan empleando nanomateriales como alternativas ya sea para la detección, mejorando el monitoreo de la calidad del agua, o su tratatamiento (desinfección o eliminación de sustancias tóxicas). Las ventajas del uso de estos materiales parecen superar sus posibles riesgos, aunque será muy importante dar seguimiento a la evolución del conocimiento alrededor de los usos y riesgos de su aplicación a mayor escala. A pesar de que ya se cuenta con algunas aplicaciones comerciales, los retos que se tienen son aún importantes y nuevas aportaciones nos irán permitiendo aprovechar al máximo las extraordinarias propiedades que nos ofrece esta vertiente científicotecnológica. 


\section{Conclusiones}

El surgimiento de los nanomateriales ofrece una alternativa para enfrentar los retos que representa la contaminación de nuestro ambiente. Este artículo resume, de manera muy breve, información sobre algunos nanomateriales aplicados al tratamiento de agua, muestra el mecanismo que explica el funcionamiento de los nanocatalizadores fotocatalíticos y algunos ejemplos de su aplicación.

El uso a gran escala de estos últimos materiales, aún no es posible debido a diversas limitaciones que aún existen en el ámbito del aprovechamiento de la energía solar, en el campo de desarrollo de reactores y algunos otros aspectos como son encontrar la manera de manejarlos y reutilizarlos fácilmente. No hay que olvidar que paralelamente a sus beneficios, es posible que su uso represente un riesgo, por lo que la investigación sobre la aplicación de estos materiales es un tema que estará vigente en las próximas décadas.

\section{Bibliografia}

Brame J., Li Q, Alvarez P. J.J. (2011). Nanotechnology-enabled water treatment and reuse: emerging opportunities and challenges for developing countries. Trends in Food Science \& Technology, 22(11): 618-624.

Choi W., Termin A. y Hoffmann M. R. (1994). The role of metal ion dopants in quantum-sized $\mathrm{TiO}_{2}$ : Correlation between photoreactivity and charge carrier recombination dynamics. J. Phys. Chem. 98: 13669-13679.

Crane R.A., Scott T.B. (2012). Nanoscale zero-valent iron: Future prospects for an emerging water treatment technology. Journal of Hazardous Materials, vols. 211-212: 112-125.

Dunlop P. S. M., McMurray T. A., Hamilton J. W. J. y Byrne J. A. (2008). Photocatalytic inactivation of Clostridium perfringens spores on $\mathrm{TiO}_{2}$ electrodes. Journal of Photochemistry and Photobiology A, vol. 196, núm. 1: 113-119.

Ede S., Hafner L., Dunlop P., Byrne J. y Will G. (2012). Photocatalytic disinfection of bacterial pollutants using suspended and immobilized $\mathrm{TiO}_{2}$ powders. Photochemistry and Photobiology, vol. 88, núm. 3: 728-735.

Gelover S., Gómez L. A., Reyes K. y Leal Ma. T. (2006). Practical demonstration of water disinfection using $\mathrm{TiO}_{2}$ films and sunlight. Water Research, 40: 32743280.

Gelover S., Mondragón P. y Jiménez G. A. (2004). Titanium dioxide sol-gel deposited over glass and its application as a photocatalyst for water decontamination. J. Photochem. Photobiol. A: Chem, 165 (1-3): 241-246.

Jiang D., Zhang S., Zhao H. (2007). Photocatalytic degradation characteristics of different organic compounds at $\mathrm{TiO}_{2}$ nanoporous film electrodes with mixed anatase/rutile phases. Environ Sci Technol, enero 1; 41(1): 303-8. 
Kamat P. V. (1997). Composite semiconductor nanoclusters. Semiconductor Nanoclusters - Physical, Prashant V. Kamat and Dan Meisel (eds.) Chemical, and Catalytic Aspects. Studies in Surface Science and Catalysis. Series, vol. 103: 1-474.

Liga M. V., Maguire-Boyle S. J., Jafry H. R., Barron A. R., Li Q. (2013). Silica decorated $\mathrm{TiO}_{2}$ for virus inactivation in drinking water-simple synthesis method and mechanisms of enhanced inactivation kinetics. Environ Sci Technol, junio 18; 47(12): 6463-70.

Lonnen J., Kilvington S., Kehoe S. C., Al-Touati F., McGuigan K. G. (2005). Solar and photocatalytic disinfection of protozoan, fungal and bacterial microbes in drinking water. Water Res, 39(5): 877-83.

Martínez A. I., Acosta D. López A. (2003). Efecto del contenido de Sn sobre las propiedades físicas de películas delgadas de $\mathrm{TiO}_{2}$. Superficies y Vacío, 16(1): 5-9, marzo.

Pradeep T. (2009). Noble metal nanoparticles for water purification: A critical review. Thin Solid Films, 517(24): 6441-6478.

Sang X., Phan T. G., Sugihara S., Yagyu F., Okitsu S., Maneekarn N., Müller W. E., Ushijima H. (2007). Photocatalytic inactivation of diarrheal viruses by visiblelight-catalytic titanium dioxide. Clin Lab., 53(7-8): 413-21.

Sharma Y. C., Srivastava V., Singh V. K, Kaul S. N. y Wen C. H. (2009). Nano-adsorbents for the removal of metallic pollutants from water and wastewater. Environmental Technology, 30(6): 583-609.

Sunada, K., Watanabe, T., Hashimoto, K. (2003). Bactericidal activity of copper-deposited $\mathrm{TiO}_{2}$ thin film under weak UV light illumination. Environ. Sci. Technol, 37: 4785-4789.

Tada, H., Kiyonaga, T., Naya, S. (2009). Rational design and applications of highly efficient reaction systems photocatalyzed by noble metal nanoparticle-loaded titanium (IV) dioxide. Chem. Soc. Rev., 38, 1849-1858.

Theron J., Walker J. A. y Cloete T. E. (2008). Nanotechnology and water treatment: applications and emerging opportunities. Critical Reviews in Microbiology, 34(1): 43-69.

Yang D., Sarina S., Zhu H., Liu H., Zheng Z., Xie M., Smith S. V. y Komarneni S. (2011). Capture of radioactive cesium and iodide ions from water by using titanate nanofibers and nanotubes. Angewandte Chemie International Edition, 50(45): 10594-10598.

Zaleska A. (2008). Doped-TiO ${ }_{2}$ : A Review. Recent Patents on Engineering, 2: 157-164. 